\title{
The polySTRAND model of flow-induced nucleation in polymers
}

\author{
Daniel J. Read ${ }^{1}$, Claire McIlroy ${ }^{2,3}$, Chinmay Das ${ }^{1}$, Oliver G. Harlen ${ }^{1}$, and Richard S. Graham ${ }^{3}$ \\ ${ }^{1}$ School of Mathematics, University of Leeds, Leeds LS2 9JT, UK. \\ ${ }^{2}$ School of Mathematics and Physics, University of Lincoln, Lincoln, LN6 7TS, UK. \\ ${ }^{3}$ School of Mathematical Sciences, University of Nottingham, Nottingham NG7 2RD, UK.
}

(Dated: April 1, 2020)

\begin{abstract}
We develop a thermodynamic continuum-level model, polySTRAND, for flow-induced nucleation in polymers suitable for use in computational process modelling. The model's molecular origins ensure it accounts properly for flow and nucleation dynamics of polydisperse systems and can be extended to include effects of exhaustion of highly deformed chains and nucleus roughness. It captures variations with the key processing parameters, flow rate, temperature and molecular weight distribution. Under strong flow, long chains are over-represented within the nucleus, leading to super-exponential nucleation rate growth with shear rate as seen in experiments.
\end{abstract}

PACS numbers: $64.70 . \mathrm{km}, 64.60 . \mathrm{qe}, 83.80 . \mathrm{Sg}$

Crystal nucleation in polymers is strongly enhanced by flow $[1,2]$, and this flow-induced crystallisation (FIC) is a prominent unsolved problem in polymer physics. FIC is an externally driven, non-equilibrium phase transition that controls crystallisation in industrial polymer processing. Hence, a molecular understanding of FIC would enable design of semi-crystalline products by tailoring processing conditions. This requires a quantitative model for the key control parameters of temperature, flow rate and molecular weight distribution (MWD).

Flow rapidly accelerates the crystallisation kinetics through an enhanced nucleation rate, $\dot{N}$ (see Fig.1). Beyond this, even stronger flow produces the aligned shish kebab morphology [2-4]. Both nucleation and alignment increase with flow rate, strain, molecular weight and long chain concentration [3-12]. Despite substantial experimental progress, key obstacles remain [13]: (1) nucleation events are rare, rapid and spatially localised, so experiments make observations long after critical nucleation has occurred; (2) synthesising monodisperse crystallizable polymers is difficult so most FIC experiments involve broad MWDs. Under flow, such melts have a wide range of chain deformation, and nucleation results from the cooperation of many chains of widely differing deformation and concentration. Polydispersity is ubiquitous in experiments and processing, but obscures the central physics of flow-induced nucleation (FIN).

Molecular dynamics (MD) simulations can complement experiments. Recent simulations have resolved individual nucleation events from monodisperse chains, to quantify FIN [14-19]. Simulations of 150-carbon polyethylene [15], showed that the Kuhn segment nematic order, $P_{2, K}$, is the key parameter for FIN. However, MD simulations cannot reach the MWD and undercooling required to directly model experiments or industrial processing. At much higher coarse-graining, continuum models comprise deterministic differential equations [2024]. These can access long spatiotemporal scales but currently struggle with polydispersity: they use insuffi-

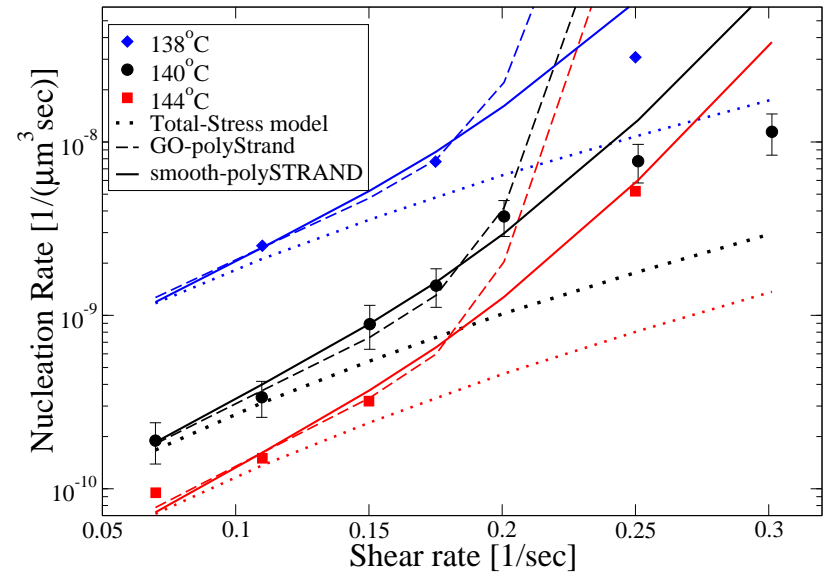

FIG. 1. Steady-state $\dot{N}$ measurements against $\dot{\gamma}$ for an iPP melt[9] and the polySTRAND model predictions. The totalstress model pre-averages chain deformation into a single species.

ciently detailed rheological models and do not account for nucleation from multiple chains with different deformations. At intermediate coarse-graining is the GO model $[25,26]$, which simulates the nucleation dynamics stochastically but uses a constitutive equation for the amorphous chain dynamics. It can access long chains and low-undercooling. Despite encouraging comparisons with experiments [25, 27], the model lacks a constitutive equation valid for polydisperse melts [25] and is too expensive for computational modelling of polymer processing [13].

We present a comprehensive resolution to the above long-standing issues by uniting multiple levels of coarsegraining, including MD, kinetic Monte Carlo and continuum modelling, to produce a computationally fast analytic model with deep molecular roots: we recalibrate the GO model to recent MD simulations [15]; we use a recent constitutive equation [28] for polydisperse melts; and we derive a novel analytic solution of the GO model $[25,26]$. This leads to a fully polydisperse model of FIN 
- the POLYdisperse STRain Accelerated Nucleation Dynamics (polySTRAND) model. We derive two sub models: GO-polySTRAND closely captures the GO model; smooth-polySTRAND captures experiments by including a nucleus roughness penalty and local exhaustion of long chains. Fig.1 summarises our main results, to be discussed in more detail below: (i) a simple average across the polymer chain population (total-stress model), which does not properly account for the effects of polydispersity, is insufficient to predict the strong acceleration of nucleation rate with $\dot{\gamma}$; (ii) polydispersity leads to enrichment of long chains in the critical nucleus, giving a superexponential dependence of the nucleation rate on shear (GO-polySTRAND); and (iii) this enrichment is limited: local exhaustion of long chains (smooth-polySTRAND) limits this super-exponential behaviour.

Model overview: In the GO model nuclei are ellipsoids of $N_{S}$ stems and $N_{T}$ monomers (Fig 2a). The quiescent nucleus potential is $U_{\text {nuc }}=-\epsilon_{B} N_{T}+\mu_{S} S\left(N_{T}, N_{S}\right)$, where $\epsilon_{B}$ is the free energy of crystallization per monomer, $\mu_{S}$ is the surface energy cost, and $S$ is the surface area. All energies are in units of $k_{B} T$. The model assumes that the flow-induced reduction in chain entropy can be subtracted from the entropy penalty for crystallization. Each chain species deforms differently so has its own attachment rate. The stem attachment $k_{s t}^{+}$and detachment $k_{s t}^{-}$rates obey $k_{s t}^{+} / k_{s t}^{-}=\phi_{i} \exp \left(-\Delta U_{\text {nuc }}+\Delta f_{i}\right)$, where $\phi_{i}$ is the melt volume fraction of species $i$ and $\Delta f_{i}$ is the change in monomer free energy from chain deformation. Existing stems can attach or detach a monomer of the same species whose volume fraction at the nucleus surface taken as 1 . Thus the ratio of rates for existing stems is identical to $k_{s t}^{+} / k_{s t}^{-}$but with $\phi_{i}=1$.
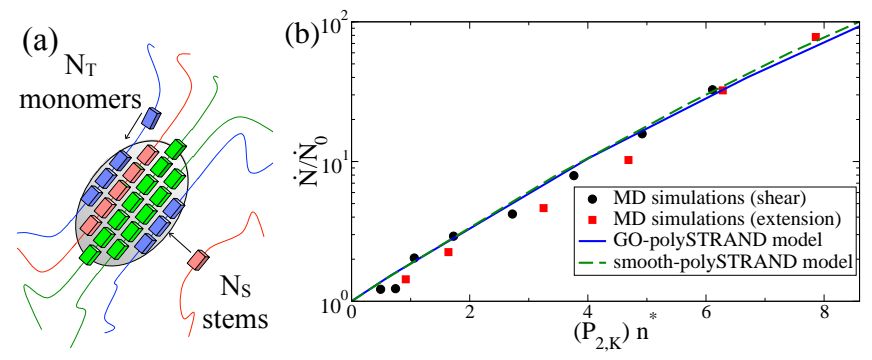

FIG. 2. (a) A nucleus in the GO model [25]. Blocks are crystallised segments, smooth lines are amorphous. Colours are chains with different deformation. Each chain forms a separate stem. (b) Nucleation rate over quiescent rate against Kuhn step nematic order for MD [15] and the ourec models. The quiescent critical nucleus, $n^{*}$, in MD is 12 Kuhn steps.

GO-polySTRAND: We derive an analytical expression for the nucleus free energy consistent with the GO model. The nucleus has stems of species $i$ with fraction $w_{i}$ (number of $i$ stems is $N_{S} w_{i}$ ) and monomers with fraction $v_{i}$ (number of $i$ monomers is $N_{T} v_{i}$ ). The nucleus entropy contains (i) the number of arrangements of $N_{S} w_{i}$ stems of each species among $N_{S}$ stems, and (ii) the number of ways to distribute $N_{T} v_{i}$ monomers among $N_{S} w_{i}$ stems. For $q=N_{S} / N_{T}$ and large $N_{S}$ and $N_{T}$ the nucleus free energy is (see Supplementary Information (SI) [29] §1):

$$
\begin{aligned}
F=N_{T} \sum_{i} & {\left[q w_{i}\left(2 \log w_{i}-\log \phi_{i}\right)-v_{i} \log v_{i}\right.} \\
& \left.+\left(v_{i}-q w_{i}\right) \log \left(v_{i}-q w_{i}\right)-v_{i} \Delta f_{i}\right] \\
& +N_{S} \log q-\epsilon_{B} N_{T}+\mu_{S} S\left(N_{T}, N_{S}\right),
\end{aligned}
$$

Minimisation over $\left\{w_{i}, v_{i}\right\}$ with $\sum_{i} w_{i}=\sum_{i} v_{i}=1$ yields

$$
w_{i}=\frac{B \phi_{i} \exp \left(\Delta f_{i}\right)}{1-A \exp \left(\Delta f_{i}\right)}, v_{i}=\frac{B q \phi_{i} \exp \left(\Delta f_{i}\right)}{\left[1-A \exp \left(\Delta f_{i}\right)\right]^{2}},
$$

where $A$ and $B$ are Lagrange multipliers, determined by,

$$
\sum_{i} \frac{q \phi_{i} \exp \left(\Delta f_{i}\right)}{\left(1-A \exp \left(\Delta f_{i}\right)\right)^{2}}=\sum_{i} \frac{\phi_{i} \exp \left(\Delta f_{i}\right)}{1-A \exp \left(\Delta f_{i}\right)} ; \quad B=\frac{1}{\sum_{i} \frac{\phi_{i} \exp \left(\Delta f_{i}\right)}{1-A \exp \left(\Delta f_{i}\right)}}
$$

Here, the first equation is solved numerically for $A$ (there is always a suitable $A$ such that the denominators are positive) and the second gives $B$ directly. The GOpolySTRAND is computationally fast, only requiring numerical solution of the equation for $A$. The model also enables addition of further physics without costly microscopic simulations.

Eq.1 is projected onto $N_{T}$ alone by minimising over $N_{S}$ and approximating fluctuations in $N_{S}$ via $\partial^{2} F / \partial N_{S}^{2}$ (see SI [29] §1.3). The same algorithm computes the quiescent free energy, leading to the change in free energy $\Delta F_{\mathrm{pS}}=F_{\mathrm{pS}}^{\mathrm{q}}-F_{\mathrm{pS}}^{\text {flow }}$, cancelling some of the error from finite $N_{S}$. The exact calculation of the quiescent barrier from ref [27] provides the final free energy barrier via $F_{\text {final }}=F_{\mathrm{GO}}^{\mathrm{q}}-\Delta F_{\mathrm{pS}}$. Barriers from this method agree very closely with GO simulations [27] (see SI [29] §1.4). We calculate $\dot{N}$ by solving a 1D Kramer's problem [30], which accurately predicts $\dot{N}$ from GO simulations for a given nucleation barrier [27] (see SI [29] §3).

Next, we require $\left\{\Delta f_{i}\right\}$. In MD simulations [15] of two-entanglement chains, $\dot{N}$ grows exponentially with the Kuhn segment nematic order $P_{2, K}$. Here, head-totail symmetry means there is effectively one species. For a single species at moderate $\Delta f_{i}$, polySTRAND predicts $\dot{N} \propto \exp \left(\Delta f n^{*}\right)$, where $n^{*}$ is the quiescent critical nucleus size. Hence taking $\Delta f=\Gamma P_{2, K}$, with $\Gamma$ a constant, gives the exponential behaviour seen in $\mathrm{MD}$ and $\Gamma=0.65$ gives quantitative agreement (Fig $2 \mathrm{~b}$ ). A polydisperse melt requires $P_{2, K}$ for each chain length. Hence we use the Rolie-Double-Poly (RDP) constitutive equation [28] to compute chain configuration tensors $\mathbf{A}$ under flow for each species. The MWD is discretized into $N$ species, with species $i$ having fraction $\phi_{i}$ and $Z_{i}$ entanglements. The RDP computes $\mathbf{A}_{i j}$, the effect of species $j$ on species $i$, via $N^{2}$ coupled differential equations. The overall configuration for species $i$ is $\mathbf{A}_{i}=\sum_{j} \phi_{j} \mathbf{A}_{i j}$. The order parameter for species $i$ is $P_{2, K, i}=\Lambda_{\max } / N_{e}$, where $\Lambda_{\max }$ is 
the largest eigenvalue of $\mathbf{A}_{i}-\mathbf{I}$ and $N_{e}$ is the Kuhn steps per entanglement. The RDP enables us to resolve $P_{2, K}$ for each chain length and then use the polySTRAND model to compute their co-operative effect on nucleation. We also compare this to a simplified 'total-stress' model where the total melt stress $\left(\mathbf{A}_{T}=\sum_{i} \phi_{i} \mathbf{A}_{i}\right)$ is used to compute a pre-averaged single species $P_{2, K, a v}$.

Fig 1 shows steady-shear $\dot{N}$ measurements from a polydisperse isotactic polypropylene (iPP) [9]. The totalstress model predicts $\dot{N}$ is, at most, exponential in the shear rate, $\dot{\gamma}$, since $P_{2, K, a v}$ grows sublinearly with $\dot{\gamma}$ for a shear thinning fluid. In contrast, the GO-polySTRAND captures the upward curvature in the data at all temperatures. This arises directly from enrichment of highlydeformed chains in the nucleus (Eq 2 indicates strong enrichment in the fraction $w_{i}$ of stems with large $\Delta f_{i}$, i.e. oriented chains, predisposed to nucleation, and further enrichment in $v_{i}$ ); this long-chain enrichment, as well as a flow model capable of predicting individual chain species dynamics, is indispensable to model $\dot{N}$.

Long-chain enrichment apparently contradicts scattering data by Kimata et al. [3], showing no overrepresentation of long chains in shish structures. However, our model predicts enrichment only in pre-critical nuclei. Post-nucleation growth is thermodynamically favourable so recruits all chains equally, giving the melt distribution in well-developed crystals. Thus enrichment strongly influences $\dot{N}$, but will not be observed in larger crystals. Indeed Kimata et al. observed that the long chains catalyse the recruitment of other chains into shish. To quantitatively understand shish formation, we require a detailed understanding of polydispersity in point-like nucleation, as provided by the polySTRAND model.

The model parameters required for experimental comparison fall into four classes: (i) material (Kuhn step density $\rho_{K}$ and crystal growth rate $G_{c}$ ) and (ii) rheological parameters (entanglement molecular weight $M_{e}$, Rouse time of an entanglement segment $\tau_{e}$ and $N_{e}$ ): these are obtained from the literature or linear rheological measurements; (iii) quiescent nucleation parameters (monomer attachment time $\tau_{0}$, and the quiescent nucleation barrier, specified by $n^{*}$ and the quiescent barrier height $\Delta F_{q}^{*}$, or equivalently $\mu_{S}$ and $\epsilon_{B}$ ) which are obtained from the quiescent crystallisation measurements; and (iv) a single, order 1 , FIC parameter $(\Gamma)$, which is fitted to low-shear FIC experiments (see SI [29] §4). All parameters are independent of MWD, except for $\tau_{e}$ which depends weakly on MWD. Only certain parameters $\left(\tau_{e}, n^{*}\right.$ and $\left.\Delta F_{q}^{*}\right)$ depend on temperature. To model the data in Fig. 1 at $140^{\circ} \mathrm{C}$, we took the material and rheological parameters from ref [25]: $M_{e}=4.4 \mathrm{~kg} \mathrm{~mol}^{-1}$, $\tau_{e}=90 \mathrm{~ns}, N_{e}=25, \rho_{K}=2.7 \times 10^{9} \mu \mathrm{m}^{-3}, \tau_{0}=0.76 \mathrm{~ns}$ and $n^{*}=1000$ monomers $\left(G_{c}\right.$ is not required to model the nucleation rate). We modeled the MWD as a generalized exponential distribution, as implemented in the RepTate software [31] with parameters selected to give the reported $M_{w}$ and $M_{n}$. We adjusted the quiescent barrier height $\Delta F_{q}^{*}$ and $\Gamma$ to capture the lowest two shear rates at $140^{\circ} \mathrm{C}$, obtaining $65.0 k_{B} T$ and 4.3 , respectively. Finally, we slightly enhanced the high-molecular weight tail to capture the curvature of the $140^{\circ} \mathrm{C}$ experiments, adding a mode of mass $1.9 \times 10^{5} \mathrm{~kg} \mathrm{~mol}^{-1}$ at $\phi=0.03 \%$. To model the other temperatures we adjusted only $\epsilon_{B}$ to capture the lowest $\dot{\gamma}$, obtaining $\Delta F_{q}^{*}$ of 63.8 and $66.7 k_{B} T$ at 130 , and $144^{\circ} \mathrm{C}$, respectively.

Smooth-polySTRAND: The GO-polySTRAND overpredicts the curvature at high $\dot{\gamma}$ (Fig.1) suggesting a bound to the enrichment of deformed chains. This may be because nucleus growth allows insufficient time to draw stems from an infinite supply in the surrounding melt. Instead stems are drawn from a small region around the nucleus. Also, the GO-polySTRAND model allows the nucleus to be composed of stems of widely different lengths: this is unfavourable, as only stems of similar length benefit fully from crystallization. We account for these as follows. (1) A growing nucleus draws $N_{S}$ stems at fraction $w_{i}$, from a limited number $Q_{S}$ of stems with initial fraction $\left\{\phi_{i}\right\}$, leaving $Q_{S}-N_{S}$ remaining stems at fraction $\theta_{i}$, such that $\left(Q_{S}-N_{S}\right) \theta_{i}+N_{S} w_{i}=$ $Q_{S} \phi_{i}$. We replace the term $-N_{S} \sum_{i} w_{i} \log \phi_{i}$ in Eq. 1 with $\sum_{i}\left[\left(Q_{S}-N_{S}\right) \theta_{i} \log \theta_{i}-Q_{S} \phi_{i} \log \phi_{i}\right]$, the change in stem translation entropy between the initial melt $\left(Q_{s}\right.$ stems at fraction $\left.\phi_{i}\right)$ and the final depleted melt $\left(Q_{s}-N_{s}\right.$ stems at fraction $\left.\theta_{i}\right)$. (2) We penalise deviations of the stem length $l$ (in monomers) from the average $L=N_{T} / N_{S}$, via an energy $\frac{1}{2} \kappa(l-L)^{2}$. We now proceed as with the GO-polySTRAND model by deriving the nucleus free energy. A partition sum over stem lengths at fixed $N_{T}$ gives this to be (see SI [29] 22 ):

$$
\begin{aligned}
F & =\sum_{i}\left[\left(Q_{S}-N_{S}\right) \theta_{i} \log \theta_{i}-Q_{S} \phi_{i} \log \phi_{i}+N_{S} w_{i} \log w_{i}\right] \\
& -N_{T} \mathrm{E}(\Delta f)-\left(N_{S} / 2 \kappa\right) \operatorname{Var}(\Delta f)+\frac{1}{2} \log N_{S} \\
& -\frac{1}{2}\left(N_{S}-1\right) \log (2 \pi / \kappa)-\epsilon_{B} N_{T}+\mu_{S} S\left(N_{T}, N_{S}\right)
\end{aligned}
$$

where $\mathrm{E}(\Delta f)$ and $\operatorname{Var}(\Delta f)$ are the mean and variance of $\Delta f_{i}$ over distribution $w_{i}$. Minimisation over $\left\{w_{i}\right\}$ gives the chemical equilibrium of stems between the nucleus and the locally surrounding melt:

$$
w_{i}=\frac{Q_{S} \phi_{i}}{N_{S}+B\left(Q_{S}-N_{S}\right) \exp \left((P / \kappa-L) \Delta f_{i}-\Delta f_{i}^{2} / 2 \kappa\right)},
$$

where $B$ and $P$ are determined from $\sum_{i} w_{i}=1$ and $\sum_{i} w_{i} \Delta f_{i}=P$. We use $Q_{S}=Q_{S 0} N_{S}$, so the number of available stems grows with $N_{S}$, and $\kappa=\kappa_{0}+1 / L^{2}$, so $\kappa$ is sufficiently large for all relevant nucleus shapes. This adds two FIC parameters, $Q_{S 0}$ and $\kappa_{0}$, which are fitted to experiments in Fig 1. The final barrier is computed directly, as we have no exact solution to the quiescent problem as in GO-polySTRAND, and the nucleation rate is calculated as before (see SI [29] §3). Fig. 1 shows improved agreement at high $\dot{\gamma}$ due to local exhaustion of 
long chains; here we fitted $Q_{S 0}=30$ and $\kappa_{0}=0.1$. To capture the data, we also needed to slightly increase the mass of the extra high-molecular weight mode to $3.0 \times 10^{5}$ $\mathrm{kg} \mathrm{mol}{ }^{-1}$.

We verify the model for varying MWD using the experiments of Acierno et al. [32], who studied a range of isotactic poly-1-butene (iPB) melts (see Fig 3). These FIC experiments applied a constant $\dot{\gamma}$ for time $t_{s}$, so that $\dot{\gamma} t_{s}=60$, and recorded the half-time of the resulting turbidity evolution, $t_{1 / 2}$, all at $103^{\circ} \mathrm{C}$. In all cases $t_{s} \ll t_{1 / 2}$. Such measurements are invariably affected by heterogeneous nucleation due to embedded particles that provide a favourable surface for nucleation [18, 19]. To model these data we assume: the heterogeneous nucleation density, $N_{0}$, at $103^{\circ} \mathrm{C}$, varies by sample; quiescent homogeneous nucleation and post-shear nucleation are negligible; shear creates $\dot{N}_{\dot{\gamma}} t_{s}$ extra nuclei, where $\dot{N}_{\dot{\gamma}}$ is the steady-state FIN rate from the smooth-polySTRAND model; the FIN barrier at zero shear (characterized by $\Delta F_{q}^{*}$ and $n^{*}$ ) and all other crystallisation parameters do not vary between samples; and $t_{1 / 2}$ occurs when the crystal fraction reaches $\phi_{c}=10 \%$, although our conclusions are insensitive to the exact value. Thus we computed $t_{1 / 2}$ by combining $\dot{N}_{\dot{\gamma}} t_{s}$ and $N_{0}$ in the Schneider rate equations [33]. We obtained model parameters as follows. Rheological parameters were fitted to linear rheological measurements (see SI [29] §4.3). The growth rate for $\mathrm{iPB}$ at $103^{\circ} \mathrm{C}$ [34] is $G=0.063 \mu \mathrm{m} / \mathrm{sec}$. We computed $\rho_{K}=5.3 \times 10^{8} \mu \mathrm{m}^{-3}$ from the melt density and $\tau_{0}=0.31 \mu \mathrm{s}$ by projecting $\tau_{e}$ to the Kuhn step lengthscale. The quiescent $t_{1 / 2}$ determines $N_{0}$, giving values of $1.3,8.3,12$ and $37 \times 10^{-12} \mu \mathrm{m}^{-3}$, respectively, for iPB116, 177, 295 and 398. We took $Q_{S 0}=30$ and $\kappa_{0}=0.1$ from our iPP modelling above. We fitted the remaining parameters, $\Gamma$ and $\Delta F_{q}^{*}$ and $n^{*}$, to the FIC data for iPB166 only, giving $1.3,68 k_{B} T$ and 540 , respectively. In summary, all samples required fitting to linear rheology and quiescent crystallisation data, while a single FIC parameter $\Gamma$ was fitted to FIC data for the lowest molecular weight only. Fig 3 shows the model predicts $t_{1 / 2}$, for all samples, whenever there are isotropic crystals, up to the emergence of rod-like crystals, successfully predicting the effect of varying MWD. Where there are entirely rod-like crystals the model generally predicts a lower $t_{1 / 2}$ than experiments. This could be due to overprediction of $\dot{N}$ at high $\dot{\gamma}$, as in Fig 1 . Also absent from our model is the slower crystallisation kinetics of rod-like crystals, compared to spherulites, due to their lower growth dimension. The model overpredicts $t_{1 / 2}$ for the highest $M_{w}$ at the lowest $\dot{\gamma}$. Here, the nucleation density is very high and $t_{s}$ is long, suggesting that crystallisation during the flow may be non-negligible.

Conclusions: Using systematic multiscale modelling we derived a highly tractable model of FIN with deeprooted molecular origins. We used MD [15] as a highresolution pseudo-experiment to extract the key physics

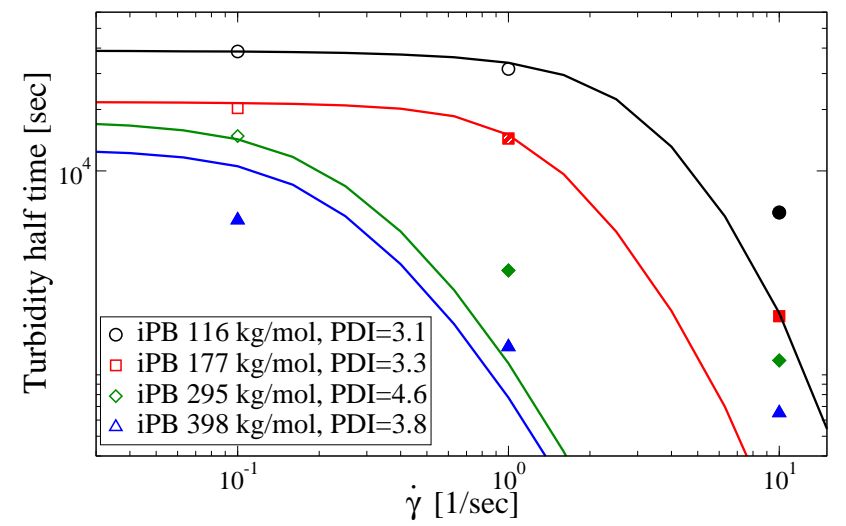

FIG. 3. Experiments [32] and the smooth-polySTRAND for $t_{1 / 2}$ after a shear of $\gamma=60$ for iPB. The legend shows $M_{w}$ and $M_{w} / M_{n}$ (PDI). The open, closed and shaded symbols indicate spherical, rod and mixed morphologies, respectively.

of FIN; we extended the spatiotemporal range with highly coarse-grained kinetic Monte Carlo simulations $[25,26]$; and, via a low-dimensional projection and thermodynamic modelling, we produced a rapidly solvable model for FIN. Our coordination of these techniques provides a road-map for problems with very widely separated spatiotemporal scales, common throughout molecular physics. Our thermodynamic modelling provides a flexible, analytic and broadly-applicable framework to capture general anisotropic nucleation under external fields. We illustrated this flexibility by adding local exhaustion of long chains and a penalty for nucleus roughness. Future extensions of this approach may produce a fully non-equilibrium treatment of diffusion and concentration gradients around the nucleus. Using a recent nonlinear flow model, we account properly for the dynamics of each chain species, not just the total stress. Our FIN model makes successful quantitative predictions for variations with flow rate, temperature and molecular weight distribution, which are key processing control parameters. Our model predicts the enrichment of long chains during nucleation. A signature of this enrichment, seen in experiments, is super-exponential growth of $\dot{N}$ with $\dot{\gamma}$. This super-exponential effect vanishes in single species models. Our FIN model is nearly analytic, so is suitable for computational modelling of polymer processing. The models in this letter are available in the Reptate software $[31]$

We thank Victor Boudara for useful discussions and Quan Chen for iPB rheology. We acknowledge funding from the EPSRC (EP/P005403/1) and the Royal Commission for the Exhibition of 1851 and access to University of Nottingham High Performance Computing and HPC Midlands+ (EPSRC grant No. EP/P020232/1). 
[1] A. Keller and H. W. Kolnaar, in Processing of Polymers, edited by H. Meijer (Wiley, Weinheim, 1997).

[2] F. L. Binsbergen, Nature 211, 516 (1966).

[3] S. Kimata et al., Science 316, 1014 (2007).

[4] O. O. Mykhaylyk et al., Macromolecules 41, 1901 (2008).

[5] M. Seki et al., Macromolecules 35, 2583 (2002).

[6] G. Kumaraswamy, A. M. Issaian, and J. A. Kornfield, Macromolecules 32, 7537 (1999).

[7] M. Stadlbauer et al., J. Rheol. 48, 631 (2004).

[8] E. E. Bischoff White, H. H. Winter, and J. P. Rothstein, Rheol Acta 51, 303 (2012).

[9] R. Pantani et al., Macromolecules 43, 9030 (2010).

[10] F. G. Hamad, R. H. Colby, and S. T. Milner, Macromolecules 48, 3725 (2015).

[11] Z. Wang et al., Sci Rep 6, 32968 (2016).

[12] K. Cui et al., Chem. Rev. 118, 1840 (2018).

[13] R. S. Graham, J Rheol 63, 203 (2019).

[14] D. A. Nicholson and G. C. Rutledge, J. Chem. Phys. 145, 244903 (2016).

[15] D. A. Nicholson and G. C. Rutledge, Journal of Rheology 63, 465 (2019).

[16] M. Anwar, J. T. Berryman, and T. Schilling, J. Chem. Phys. 141, 124910 (2014).

[17] M. Anwar and R. S. Graham, J. Chem. Phys. 150, 084905 (2019).

[18] A. Jabbarzadeh, Nanomaterials 9 (2019).

[19] A. Jabbarzadeh and B. Halfina, Nanoscale Advances 1, 4704 (2019).

[20] A. Doufas, I. Dairanieh, and A. McHugh, Journal of
Rheology 43, 85 (1999).

[21] S. Coppola et al., Polymer 45, 3249 (2004).

[22] R. J. A. Steenbakkers and G. W. M. Peters, J Rheol 55, 401 (2011).

[23] P. C. Roozemond et al., J Rheol 59, 613 (2015).

[24] C. McIlroy and R. S. Graham, Additive Manufacturing 24, 323 (2018).

[25] R. S. Graham and P. D. Olmsted, Phys Rev Lett 103, 115702 (2009).

[26] R. S. Graham and P. D. Olmsted, Faraday Discuss 144, $71(2010)$.

[27] K. Jolley and R. S. Graham, Rheol.Acta 52, 271 (2013).

[28] V. A. H. Boudara et al., Journal of Rheology 63, 71 (2019).

[29] See Supplementary Material [url] for full details of the model derivation and comparison to experiments, which includes refs [35-37].

[30] M. J. Hamer, J. A. D. Wattis, and R. S. Graham, J Non-Newton Fluid Mech 165, 1294 (2010).

[31] Available at http://reptate.readthedocs.io.

[32] S. Acierno et al., Rheologica Acta 42, 243 (2003).

[33] W. Schneider, A. Köppl, and J. Berger, Int. Polym. Proc. II 3, 151 (1988).

[34] M. Yamashita and S. Ueno, Crystal Research and Technology 42, 1222 (2007).

[35] C. Das, N. J. Inkson, D. J. Read, M. A. Kelmanson, and T. C. B. McLeish, J. Rheol. 50, 207 (2006).

[36] K. Jolley and R. S. Graham, J Chem Phys 134, 164901 (2011).

[37] C. Liu, P. Liu, Q. Chen, B. Du, and Z. Wang, Journal of Rheology 63, 167 (2019). 